\title{
Nuclear Alpha Decay in Field of Synchrotron Radiation
}

\author{
Kopytin Igor Vasilievich
}

Department Theoretical Physics, Voronezh State University, Voronezh, Russia

Email address:

i-kopytin@yandex.ru

\section{To cite this article:}

Kopytin Igor Vasilievich. Nuclear Alpha Decay in Field of Synchrotron Radiation. American Journal of Physics and Applications. Vol. 7, No. 3, 2019, pp. 89-92. doi: 10.11648/j.ajpa.20190703.15

Received: April 11, 2019; Accepted: May 10, 2019; Published: July 4, 2019

\begin{abstract}
We investigated before the possibility of modifying alpha decay rate by the influence of a laser radiation upon a nucleus. We showed that the laser radiation with the extreme achievable intensity slightly modifies the total rate of alpha decay. A different result may be probably obtained if it use synchrotron radiation for the irradiation of an alpha-active nucleus. At present, synchrotron radiation from the third generation synchrotrons has high brilliance, the photon energy may reach 200$300 \mathrm{keV}$ and, in the future, it may be larger. These energies are comparable with nuclear ones and the effect from the influence of the synchrotron radiation upon alpha decay could be more significant. As it turned out, the change of the alpha decay rate of ${ }^{238} \mathrm{U}$ isotope into the synchrotron radiation field from the third generation synchrotrons will be negligible.
\end{abstract}

Keywords: Alpha Decay, Synchrotron Radiation, Third Generation Synchrotron, ${ }^{238}$ U Isotope, Alpha Decay Rate

\section{Introduction}

The fundamental problem of external influence on various physical processes in a substance, as well as in its individual structural elements (molecules, atoms and atomic nuclei), is one of the most pressing problems of modern physics. This is due to the value of the physical information obtained about the properties of the objects of research, about new phenomena and the ability to manage them. In leading research centers, more and more powerful sources of electromagnetic radiation and particle accelerators appear. It also contributes to the development of this direction.

In the physics of atoms and molecules, many advances were associated with the effect of strong laser fields on their characteristics. It is studied the multiphoton ionization of atoms, the generation of high harmonics, the many-electron tunneling effect, the acceleration of electrons in a plasma to several $\mathrm{GeV}$, and others (see, for example, the review paper [1], as well as our work [2], which provides links to reviews of recent years in this region). The use of electromagnetic radiation from synchrotrons of the first and second generations also make a contribution to the substantial progress of physics, in particular, in studies of condensed matter and other objects (see, for example, the book [3] and references therein).

Atomic nuclei as an object of this line of research are still an exception. All attempts to influence on nuclear processes by powerful optical and soft X-ray radiation, if the latter were not caused only by rearrangement of the electron shell of an atom, were unsuccessful (review of studies on the effects of laser radiation on nuclear beta decay is given, for example, in paper [4] ). Recently, however, there may be changes in this area. Perhaps great prospects in the field of external influences on the physical processes in atomic nuclei will open up in connection with the commissioning of thirdgeneration synchrotrons at research centers in a number of countries (USA, Japan, Germany, and Russia). Already there are large storage rings APS (Advanced Photon Source) with an energy of $7 \mathrm{GeV}$ (USA) and SPRING-8 (Super Photon Ring) with an energy of $8 \mathrm{GeV}$ (Japan). In the storage ring ESRF (European Synchrotron Radiation Facility) with an electron energy of $6 \mathrm{GeV}$ (Grenoble, France), due to the built-in undulators in it, it was possible to increase the brightness of synchrotron radiation (SR) by two orders of magnitude. The storage rings of 3rd generation improve not only radiation parameters such as flux and brightness, but also create the possibility of shifting the energy range of SR to a more rigid part of the spectrum, extending to nuclear ranges. Already, the energies of SR photon without a significant decrease in its intensity reach $200-300 \mathrm{keV}$, for example, at SPRING-8 in Japan. Moreover, the prospect of obtaining energy of SR to values of the order of $1 \mathrm{MeV}$ is considered further. 
The SR photon energies of several hundred $k e V$, achievable at third-generation synchrotrons, allow us to investigate the direct effect of SR on nuclear characteristics. Energy of such magnitude and high radiation power make it possible again to raise the question of the effect of electromagnetic radiation on nuclear processes, in particular, on decay processes. These are alpha and beta decays of atomic nuclei in the SR field. In this case, the values of the energy transferred to the nucleus are already quite large and comparable with nuclear ones. In this case, beta transitions, which are prohibited under natural conditions, become possible, and beta transitions, which are allowed, will be accelerated $[5,6]$. The high power of SR from thirdgeneration synchrotrons makes it possible to hope that, in contrast to beta decay stimulated by laser radiation, it will be possible to obtain the rates of the corresponding decays and their accelerations available for observation. At the very least, it will be possible to formulate the necessary requirements for the implementation of this possibility.

All of the above applies to another nuclear decay process alpha decay. The ability to change its speed by laser radiation was estimated by us earlier and it was shown that this is practically impossible [2]. In this paper, we study the possibility of influencing on the alpha decay rate by the SR field generated by third-generation synchrotrons. Specific parameters of such a radiation with a continuous spectrum (from a wiggler) were taken from site [7] for the SPRING-8 synchrotron (Japan).

\section{Evaluation of Change of Alpha Decay Rate in the Field of Synchrotron Radiation}

In paper [2], in the semi classical approximation, we obtained an expression for the correction to the rate of nuclear alpha decay in the presence of an alternating electromagnetic field. For the case of laser radiation, the dependence of the relative change in the alpha decay rate on the radiation intensity was calculated. The effects of the charge on the alpha-active nucleus and the energy of the alpha particle were also investigated. It was shown that even for theoretically limiting values of the intensity of laser radiation $\left(10^{26}-10^{28} \mathrm{~W} / \mathrm{cm}^{2}\right)$, which are so far unattainable in practice, the relative acceleration of alpha decay does not exceed a few percent. In particular, for the ${ }^{238} U$ isotope $(Z=$ $\left.92, E_{\alpha}=4.27 \mathrm{MeV}\right)$ with an intensity of laser radiation of $10^{28}$ $W / \mathrm{cm}^{2}$, this acceleration is no more than $2 \%$ (hereinafter $Z$ is the charge number of the parent nucleus; $E_{\alpha}$ is the energy of the emitted alpha particle).

Perhaps a different result will be obtained if synchrotron radiation is used to irradiate the alpha-active nucleus. As already mentioned in the Introduction, at present, SR from third-generation synchrotrons has a high brightness, and the upper limit of its continuous spectrum from a wiggler can reach 200-300 keV, and without a significant loss of radiation power in this part of the spectrum. Since these energies are already comparable in magnitude with nuclear ones, the effect of the action of the SR field on alpha decay may be more noticeable than in the case of optical radiation even from the most powerful lasers. In this section, we will perform a quantitative assessment of the effect of SR on the alpha decay rate of spherical nuclei (taking into account the possible deformation of alpha-active nuclei complicates the task).

In the direct action of synchrotron radiation on the process of alpha decay, it is conditionally possible to distinguish two components. First, the alpha particle and the daughter nucleus have charges. Consequently, an external electromagnetic field will interact with them and deform the potential barrier that the alpha particle overcomes in the decay process. Such a problem was considered in paper [2] for the case of a laser field. In this work, the well-known single-particle model of Gamow (see the paper [8]) was used to describe the process of alpha decay, and here in our estimates we take it as a basis. In this model, the probability of emission of an alpha particle with a given energy from the atomic nucleus is determined mainly by the permeability of the potential barrier caused by the nuclear and Coulomb interactions of the alpha particle and the daughter nucleus.

Secondly, the interaction of SR with the alpha particle can significantly increase its energy, since it contains photons with energies up to $300 \mathrm{keV}$ (for third-generation synchrotrons). It is feature of the process of alpha decay that distinguishes the action of synchrotron radiation from the laser. In the latter case, the energy of the emitted alpha particle is changing slightly. In the one-particle model of alpha decay (see the paper [8]), the probability of decay will increase with an increase in the alpha particle energy, since the penetrability of the potential barrier will increase. Of course, all this is conditional, since in the complete theory of the alpha decay process, both of these components form a single whole. However, in order to evaluate the effect of these effects on the rate of the alpha decay process in the SR field, we still consider them separately. If such an influence is noticeable in practically interesting cases, then it will be possible to raise a question of the development of a complete theory.

Consider first the effect of the electromagnetic field on the process of alpha decay, exploring its role in the deformation of the potential barrier. We solved such a problem in the quasiclassical approximation in paper [2]. The final expression for the coefficient $K$ (it gives the ratio of the alpha decay rates in the presence and absence of the electromagnetic field) allows us to estimate the degree of influence of the field:

$$
K=\frac{\Delta \lambda_{\alpha}(I)}{\lambda_{\alpha}^{(0)}} \approx \frac{3 \pi^{3}(Z-2)^{4} e^{10} \mu}{E_{\alpha}^{5}} I
$$

Here, $\Delta \lambda_{\alpha}(I)=\lambda_{\alpha}(I)-\lambda_{\alpha}^{(0)}, \lambda_{\alpha}(I)$ and $\lambda_{\alpha}^{(0)}$ are the alpha decay rates in the presence of an electromagnetic wave with intensity $I$ and without it, $e$ is the electron charge, $\mu$ is the reduced mass of the alpha particle. Now for strong laser 
radiation, an intensity of the order of $\left(10^{21}-10^{22}\right) \mathrm{W} / \mathrm{cm}^{2}$ has practically been reached. As was shown in [2] using the example of alpha decay of the ${ }^{238} \mathrm{U}$ isotope, even for laser intensities $I=\left(10^{26}-10^{27}\right) \mathrm{W} / \mathrm{cm}^{2}$, close to the theoretical limit, the change in the rate of alpha decay of the ${ }^{238} \mathrm{U}$ isotope did not exceed $2 \%$.

Considering the effect of synchrotron radiation, it is necessary to take into account one more difference between laser radiation and SR - the latter has no coherence. Because of this, the amplitudes of the electromagnetic field do not add up. This does not allow obtaining large values of the electric field strength (and magnetic field induction). For this reason, synchrotron radiation will not be able significantly to change the width of the barrier. Moreover, at present, the intensity of $\mathrm{SR}$, even for the third-generation of synchrotrons, is still substantially less than that of laser facilities. As a result, we can conclude that the change in the alpha decay rate due to the deformation of the potential barrier by the field of synchrotron radiation will be negligible.

In the framework of the single-particle model of alpha decay [8], we now estimate how CR can affect alpha decay, increasing the energy of the emitted alpha particle. We will consider a system consisting of a daughter nucleus with a charge (Z-2)e and an $\alpha$ particle with a charge of $2 e$. In [8], their nuclear interaction is modeled by a spherical well of depth $V_{0}$ and radius $R_{0}$. Thus, the potential energy of interaction, which determines the shape of the potential barrier, has the form

$$
V(r)=\frac{2(Z-2) e^{2}}{r} \text {, if } \mathrm{r}>R_{0} \text {, and } V(r)=-V_{0}, \text { if } \mathrm{r}>R_{0} .
$$

As usual, the wave function was presented in a standard form $\Psi(\mathbf{r})=r^{-1} u(r) Y_{l m}(\vartheta, \varphi)$, where $Y_{l m}(\vartheta, \varphi)$ is a spherical function. Neglecting the centrifugal term, which, as is well known, gives a small additive to the interaction energy, we can write the Schrödinger radial equation in the form:

$$
\left(-\frac{\hbar^{2}}{2 \mu} \frac{d^{2}}{d r^{2}}+V(r)-E_{\alpha}\right) u(r)=0
$$

This equation corresponds to a rather crude model of alpha decay and there is no need to find its exact solution. For our purposes, it suffices to find the potential barrier penetrability in the quasiclassical approximation. We have

$$
P\left(E_{\alpha}\right)=\exp \left\{-\frac{2}{\hbar} \int_{r_{1}}^{r_{2}} \sqrt{2 \mu\left[V(r)-E_{\alpha}\right]} d r\right\}
$$

where $r_{1}$ and $r_{2}$ are the turning points determined from the condition

$$
V\left(r_{1}\right)=V\left(r_{2}\right)=E_{\alpha}, r_{1}>r_{2}
$$

In our case, $r_{1}=R_{0}$ и $r_{2}=2(Z-2) e^{2} / E_{\alpha}$. Integrating in (4), taking into account (2) and (5), we obtain:

$$
P\left(E_{\alpha}\right)=\exp \left\{-\frac{4(Z-2) e^{2}}{\hbar v}\left(\pi-2 \varphi_{0}-\sin 2 \varphi_{0}\right)\right\}
$$

where $v=\sqrt{2 E_{\alpha} / \mu}$ - the speed of alpha particles, $\sin \varphi_{0}=\left[E_{\alpha} R_{0} / 2(Z-2) e^{2}\right]^{1 / 2}$.

To obtain the alpha decay rate (probability per unit time) in the Gamow model, the penetrability of the barrier should be multiplied by the "frequency" with which the alpha particle hits the "boundary" of the nucleus. In [8], it was assumed that this "frequency" is equal to $v / 2 R_{0}$, i. e. proportional to magnitude $E_{\alpha}^{1 / 2}$. There are other ways of estimating the magnitude of this factor in the single-particle model. However, we are only interested in the relative rate of alpha decay, as in the previous case, when the coefficient $K$ was calculated. Therefore, the differences in the definition of the above "frequency" will not be very significant.

\section{Results}

Using the formula (6) for the penetrability of the barrier $P$, we estimate the change in its magnitude if the alpha particle energy $E_{\alpha}$ is increasing by $200 \mathrm{keV}$ in the electromagnetic field (the photon energy of synchrotron radiation from a wiggler in the SPRING-8 synchrotron reaches the magnitude of $200 \mathrm{keV}$ without a significant decrease in its intensity). As above, we estimate the value of the coefficient $\mathrm{K}$ for the case of the alpha decay of the ${ }^{238} \mathrm{U}$ nucleus. Recall that this isotope of uranium has $Z=92$ and $E_{\alpha}=4.27 \mathrm{MeV}$. The parameter $R_{0}$ was calculated using the formula $R_{0}=1.57 \times A^{1 / 3}$ fermi ( $A$ is the mass number of the nucleus ${ }^{238} \mathrm{U}$, an offsystem unit 1 fermi $=10^{-15} \mathrm{~m}$ ), the use of which allows quite satisfactory description of alpha decay to the ground state of even-even uranium nuclei [9]. As a result, the coefficient $K=$ $P\left(E_{\alpha}=4.47 \mathrm{MeV}\right) / P\left(E_{\alpha}=4.27 \mathrm{MeV}\right)$, which shows an increase in the penetrability of the barrier with an increase in the alpha particle energy of $200 \mathrm{keV}$, is equal to 42.3 .

We estimated the growth trend of the penetrability of the potential barrier $P\left(E_{\alpha}\right)$. This estimate was made by formula (6) also for alpha particles of high energy, which have not yet been obtained for SR from active synchrotrons. The results are presented in table 1 . The values of the corresponding alpha-decay rates $\lambda_{\alpha}\left(E_{\alpha}\right)$ and their acceleration coefficients $K\left(E_{\alpha}\right)=\lambda_{\alpha}\left(E_{\alpha}\right) / \lambda_{\alpha}\left(E_{\alpha}=4.27 \mathrm{MeV}\right)$ are also given. In accordance with the work [8], these rates were calculated by the formula

$$
\lambda_{\alpha}\left(E_{\alpha}\right)=\frac{v}{2 R_{0}} P\left(E_{\alpha}\right)
$$

Table 1. Characteristics of the alpha decay of the ${ }^{238} U$ isotope, calculated for different energies of alpha particle.

\begin{tabular}{llllll}
\hline $\boldsymbol{E}_{\boldsymbol{\alpha}}, \mathbf{M e V}$ & $\boldsymbol{E}_{\gamma}, \mathrm{keV}$ & $\boldsymbol{P}\left(\boldsymbol{E}_{\boldsymbol{\alpha}}\right)$ & $\lambda_{\boldsymbol{\alpha}}\left(\boldsymbol{E}_{\boldsymbol{\alpha}}\right), \boldsymbol{s}^{-1}$ & $\boldsymbol{K}\left(\boldsymbol{E}_{\boldsymbol{\alpha}}\right)$ & $\boldsymbol{\beta}\left(\boldsymbol{E}_{\boldsymbol{\alpha}}, \boldsymbol{E}_{\gamma}\right)$ \\
\hline 4.27 & 0 & $2.0 \cdot 10^{-38}$ & $1.5 \cdot 10^{-17}$ & 1.0 & - \\
4.47 & 200 & $8.4 \cdot 10^{-37}$ & $6.3 \cdot 10^{-16}$ & 42.3 & $2.9 \cdot 10^{-28}$ \\
4.77 & 500 & $1.5 \cdot 10^{-34}$ & $1.2 \cdot 10^{-13}$ & $7.8 \cdot 10^{3}$ & $1.0 \cdot 10^{-27}$ \\
5.27 & 1000 & $3.5 \cdot 10^{-31}$ & $2.8 \cdot 10^{-10}$ & $1.9 \cdot 10^{7}$ & $2.6 \cdot 10^{-25}$ \\
\hline
\end{tabular}


As can be seen from the table 1, calculation by formulas (6) and (7) of the alpha decay probability from the ground state of the ${ }^{238} \mathrm{U}$ isotope gives a value of $1.5 \times 10^{-17} \mathrm{~s}^{-1}$. It is 3 times more than the experimental value $\left(5 \times 10^{-18} \mathrm{~s}^{-1}\right)$, however, taking into account the simplicity of the alpha decay model used by us, this result should be considered satisfactory.

As expected, an increase in alpha particle energy due to the absorption of photon of SR with maximum energy leads to a significant increase in the penetrability of the potential barrier for alpha particle and, accordingly, to an increase in the alpha decay rate. Thus, with an increase in this energy by $200 \mathrm{keV}$, the alpha decay rate increases by almost two orders of magnitude $\left(K\left(E_{\alpha}\right)=42.3\right)$ and even more it increases at high alpha-particle energies (see Table 1 for the coefficient $\left.K\left(E_{\alpha}\right)\right)$. However, this fact does not mean that such an effect may become observable. The fact is that the alpha particle, being in a potential well in an excited state, will simultaneously participate in two competing processes. One of them is alpha decay, which leads to the "leaving" of alpha particles from a potential well through a potential barrier. The other is the electromagnetic transition of an alpha particle in a potential well to a state with less energy. The last process with the emission of a photon is much more likely than the alpha decay process. Table 1 in the last column shows the coefficient $\beta\left(E_{\alpha}, E_{\gamma}\right)$, giving the ratio of the alpha decay rate with a given energy of alpha particle to the probability of its electromagnetic transition to the ground state with the corresponding energy $E_{\gamma}$. The probability of the gamma transition was calculated using the well-known Weisskopf approximate formula (see, for example, [9]) for singlephoton electrical gamma transition of nucleon in atomic nucleus. In this formula, nucleon characteristics were replaced by alpha particle characteristics (the most intense E1 gamma transitions were considered). Note that in the Gamov model, only single-photon alpha-particle transitions that are linear in intensity will be possible. Multi-photon excitations will be impossible due to the lack of coherence.

From table 1, it can be seen that alpha decay, even from states with high energy, is practically incapable of competing with electromagnetic transitions. However, using the SR, we can create conditions under which the population of the alpha-partial excited state will be maintained for a certain time $\tau$ and thus alpha decay from this state will be possible. Let us estimate the magnitude of the change in the alpha decay rate for this case. The average rate of alpha decay can be calculated by the formula.

$$
\begin{gathered}
\left.\overline{\lambda_{\alpha}\left(E_{\alpha}\right.}\right)=\lambda_{\alpha}\left(E_{\alpha}=4.27 M \ni B\right) \frac{T-\tau}{T}+\lambda_{\alpha}\left(E_{\alpha}\right) \frac{\tau}{T}= \\
=\lambda_{\alpha}\left(E_{\alpha}=4.27 M \ni B\right)\left\{1+\left[K\left(E_{\alpha}\right)-1\right] \frac{\tau}{T}\right\} .
\end{gathered}
$$

Here $T$ is the half-life for the natural alpha decay of the ${ }^{238} \mathrm{U}$ isotope with an alpha particle energy of $4.27 \mathrm{MeV}$, and the values of $\lambda_{\alpha}\left(E_{\alpha}\right)$ and $K\left(E_{\alpha}\right)$ were defined above and their magnitudes are given in Table 1 . As a result, the following expression is obtained for estimating of relative change in the alpha decay rate of the ${ }^{238} \mathrm{U}$ isotope:

$$
\Delta \lambda_{\alpha} / \lambda_{\alpha} \approx 2 \cdot 10^{-18} K\left(E_{\alpha}\right) \tau
$$

where the value of the irradiation time $\tau$ should be expressed in $s$ and the theoretical value $T=\ln 2 / \lambda\left(E_{\alpha}=4.27 \mathrm{MeV}\right)=4.6 \cdot 10^{17} \mathrm{~s}$ used. It can be shown that at reasonable values of the parameter $\tau$, changing the speed of natural alpha decay of the ${ }^{238} \mathrm{U}$ isotope by the field of synchrotron radiation is almost impossible, as in the case of laser irradiation. This can be done using formula (9) and the data from the table 1 . Only at SR photon energy of 1 $\mathrm{MeV}$, which is not yet achievable, and the total exposure time of SR to about one year $\left(1\right.$ year $\left.=3.15 \times 10^{7} \mathrm{~s}\right)$, which is also unlikely, the value $\Delta \lambda_{\alpha} / \lambda_{\alpha}$ is of the order of $10^{-3}$, which could be available for measurement.

\section{Conclusion}

The results obtained allow us to conclude that the change in the rate of the process of alpha decay by exposure to it by a field of synchrotron radiation generated even by thirdgeneration synchrotrons still lies beyond the limits of the experimental possibilities of its observation. It can also be said that so far there is no need to develop an exact theory of the process. However, with an increase in the boundary energy of the synchrotron radiation and its intensity, this problem may become relevant.

\section{References}

[1] N. B. Delone, and V. P. Krainov. Atoms in Strong Light Fields. Springer Series in Chemical Physics. Vol. 28; Springer-Verlag, Berlin, Heidelberg, New York, and Tokyo (1985).

[2] I. V. Kopytin, and A. S. Kornev. Semiclassical formula for the alpha-decay rate in an electromagnetic field. Physics of Atomic Nuclei, V. 77, No. 1, P. 53 (2014).

[3] Wiedemann H. Synchrotron Radiation. Springer (2002).

[4] I. V. Ternov, V. N. Radionov, and O. E. Dorofeev. Effect of strong electromagnetic field on beta decay. Physics of Elementary Particles and Atomic Nuclei, V. 20, part 1, P. 46 (1989).

[5] A. N. Almaliev, K. N. Karelin, and I. V. Kopytin. Acceleration of beta transition by synchrotron radiation. Proceedings of Voronezh State University. Series physics, mathematics, No 1, P. 5 (2004).

[6] I. V. Kopytin, and K. N. Karelin. Effect of synchrotron radiation on nuclear beta decay. Physics of Atomic Nuclei, V. 68, No 7, P. 1138 (2005).

[7] http://www.spring8.or.jp

[8] G. A. Gamov. Zur Quantentheorie des Atomkernes. Zs. Physik, V. 51, P. 204 (1928).

[9] Preston M. A. Physics of the nucleus. Redwood City [etc.]: Addison-Wesley Publ. Company. Palo Alto, London (1962). 